\title{
Studies of Beam Induced Dipole-Mode Signals in Accelerating Structures at the SLC*
}

\author{
Mike Seidel \\ Stanford Linear Accelerator Center, Stanford University, Stanford, CA 94309 \\ RECEIVED \\ JUL $1 / 1997$ \\ Abstract \\ OSTI
}

\begin{abstract}
Beam emittance dilution by self induced transverse fields (wakefields) in accelerating structures is a key problem in linear accelerators. To minimize the wakefield effects the beam trajectory must be precisely centered within the structures. An efficient way to achieve this is to detect beam induced microwave signals in the lowest dipole mode band and to steer the beam by minimizing these signals. This paper briefly covers some experiences from SLC S-band structures, but mainly concentrates on results of a wakefield instrumentation scheme applied to a NLC prototype X-band structure and tested with beam in the SLC linac. A beam based in-situ structure straightness measurement is shown as well as results of beam steering experiments based on phase and amplitude detection of two separated modes in the structure. After centering the beam the reduction of the wakefield was demonstrated independently by probing it with a test bunch that is deflected by the residual wakefield at a short distance behind the drive bunch.
\end{abstract}

Invited talk presented at the 1997 Particle Accelerator Conference Vancouver, B.C., Canada, May 12-16, 1997

${ }^{*}$ Work supported by Department of Energy contract DE-AC03-76SF00515. 


\section{DISCLAIMER}

This report was prepared as an account of work sponsored by an agency of the United States Government. Neither the United States Government oor any agency thereof, nor any of their employees, makes any warranty, express or implied, or essumes any legal liability or responsibility for the aceuracy, completeress, or usefulness of any information, apparatus, produch, or process divelosed or represents that its use would not infringe privately owned rights. Referesce bereis to any spe. cific commercial product, process, or service by trade name. traderasti, inanufacturet, or otherwise does not aecesenrily constitute or imply its endorsement, recommendation, or favoring by the United States Goverameat or any ageacy thereof. The views and opinions of authors expressed hereid do not necessarily state or reflect those of the United States Governmeat or any agency thereof. 


\section{DISCLAIMER}

Portions of this document may be illegible in electronic image products. Images are produced from the best available original document. 


\title{
Studies of Beam Induced Dipole-Mode Signals in Accelerating Structures at the SLC
}

\author{
M. Seidel \\ Stanford Linear Accelerator Center, Stanford University, CA 94309
}

\begin{abstract}
Beam emittance dilution by self induced transverse fields (wakefields) in accelerating structures is a key problem in linear accelerators. To minimize the wakefield effects the beam trajectory must be precisely centered within the structures. An efficient way to achieve this is to detect beam induced microwave signals in the lowest dipole mode band and to steer the beam by minimizing these signals. This paper briefly covers some experiences from SLC S-band structures, but mainly concentrates on results of a wakefield instrumentation scheme applied to a NLC prototype $\mathrm{X}$-band structure and tested with beam in the SLC linac. A beam based in-situ structure straightness measurement is shown as well as results of beam steering experiments based on phase and amplitude detection of two separated modes in the structure. After centering the beam the reduction of the wakefield was demonstrated independently by probing it with a test bunch that is deflected by the residual wakefield at a short distance behind the drive bunch.
\end{abstract}

\section{INTRODUCTION AND BASIC PRINCIPLES}

A high energy beam of charged particles that passes through an accelerating structure off-center excites asymmetric electromagnetic fields, to lowest order dipole modes, that act back on the beam. These transverse wakefields dominate the beam dynamics in linear accelerators and present the most severe limitation for the preservation of small emittances during the acceleration process. These concerns are important not only for linear colliders but also for industrial or medical applications of linear accelerators. Wakefield effects can be classified as short range effects, ie. fields that deflect the tail of a single bunch on a ps time scale, and long range effects that couple the orbits of consecutive bunches in a train. Both can lead to instabilities and emittance blow up of the beam. An historical example for the performance limiting effects of transverse wakefields is the observation of the beam-breakup instability in the SLAC Two-Mile Accelerator in 1966 [1].

Since then several powerful methods for suppressing long range wakefields have been developed. The basic approaches employ detuning and/or damping of the higher order modes (HOM's) that deflect the beam. A frequency spread of the modes is introduced by a specific variation of cell dimensions. It leads to a fast decay of the wakefield in time due to destructive interference of the modes. Damping can be achieved by several methods. In the case of the SLAC damped detuned structure [2][3] small slits in the cells couple the HOM's to manifolds that run along the structure and guide them to loads. Another way is to coat the cell irises with a lossy material as in the SBLC design [4]. The so called choke-mode cavity [5] employs integrated choke filters on each cell that reflect the fundamental mode but permit a strong damping of the higher modes.

However, the described methods cannot reduce the amplitude of the short range wakefield that is mainly determined by the iris radii of the structure cells and is therefore more or less fixed by the choice of accelerating mode frequency. As a result the wakefield scales roughly as the third power of the frequency [6]. In order to reach high gradients several new collider designs employ high frequency structures that result in enhanced wakefields. The only way to keep shortrange wakefields small is to align the beam with micron precision to the electrical center of the structure, thereby preventing the excitation of asymmetric fields. An effective approach is to detect beam induced dipole mode signals directly in the structure and minimize them by moving beam or structure. The technique of using the accelerator structure itself as a beam position monitor (BPM) is being considered in several of the presently proposed linear collider designs. The advantage of this scheme over separate BPM's is that the structure measures the beam position in the only relevant reference frame, namely with respect to the electrical center of the structure. This eliminates the need for conventional structure to BPM alignment on a $\mu \mathrm{m}$ scale.

\section{EXPERIENCES WITH THE TEN-FOOT S-BAND STRUCTURE IN THE SLC}

The SLC linac consists of roughly 900 ten-foot structures which are operated at $2.856 \mathrm{GHz}$ (S-Band). Typically the vertical emittance blowup in the linac amounts to $100 \%$ to $200 \%$ and is caused by transverse wakefields. One tries to compensate the wakefield effects by BNS damping and by empirical optimization of long orbit bumps. Otherwise one relies on beam steering using BPM information. There is some evidence from long range wakefield measurements that misalignments between structures and BPM's can occasionally reach $\mathrm{mm}$ size levels [7]. The observed emittance growth suggests rms beam-to-structure misalignments of $\approx 300 \mu \mathrm{m}$. Therefore a more precise method of determining the beam orbit with respect to the electrical center of the structure would improve the performance of the machine considerably. In the SLC runs of 1995/96 several dedicated experiments were carried out to investigate beam induced dipole mode signals in the SLC structures and to evaluate their usefulness for beam steering purposes. For these experiments the signals are detected in the standard SLC waveguide network that distributes the RF power from the klystron to the input couplers of four structures. 
The signals were analyzed with crystal detectors that integrate over the whole spectrum, with a spectrum analyzer, and with a down-mixing scheme that allows for amplitude and phase detection in connection with a strip-line BPM signal to provide a beam phase reference. Details are presented in [8]. The most important results and identified problems are summarized here briefly:

- Beam position dependent modes are detected in roughly the expected frequency range $4.1 \mathrm{GHz}$ to $4.4 \mathrm{GHz}$, however, the detailed mode spectrum is not understood well. A strong signal whose origin is not known dominates the spectrum around $4.210 \mathrm{GHz}$.

- Signals generated by either horizontal or vertical offsets are not easily distinguished, complicating their interpretation. Horizontally polarized modes will couple to the TE10 mode in the input waveguide. Vertically polarized modes could either couple to the TE20 mode or, after a slight rotation, to TE10 as well. Experimental results indicate that for the same beam offset, horizontally excited signals are 5 to 10 times stronger than vertical ones.

- The input and output couplers introduce intrinsic asymmetries in the horizontal plane that possibly shift the detected center position for certain modes systematically.

- Using both phase and amplitude detection of individual modes a position measurement resolution of $12 \mu \mathrm{m} \mathrm{rms}$ was demonstrated by correlating the signal with BPM data [8]. Fifteen BPM's were used to determine the beam orbit at the structure position for this measurement in the horizontal plane. It remains to be demonstrated that the position of minimum dipole mode excitation indeed coincides with minimum wakefield deflection.

In summary the application of dipole modes for beam steering is difficult in the SLC because the structure has no dedicated ports for HOM's, and, in addition we measure a superposition of signals from four structures. To overcome this problem one has to perform multidimensional scans in both planes, offset and angle to find a global minimum of the signal amplitude. For more detailed studies it is planned to install a special setup in sector 2 that allows to access signals from individual structures.

\section{ACCELERATOR STRUCTURE SETUP EXPERIMENT (ASSET)}

In August 1996 a prototype NLC structure was installed in the SLC beamline at the low energy end of the linac. The main goals of this experiment were to measure the longrange wakefield suppression in a damped detuned structure (DDS) and to test a wakefield instrumentation scheme, based on the above described principles of dipole mode detection. The ASSET facility [9] [10] permits to send two bunches, "drive" and "witness" through the structure at a precisely controlled time separation. The drive bunch consists of positrons, the witness bunch of electrons and their trajectories are separated by a chicane downstream of the

\begin{tabular}{lcccc}
\hline & $\begin{array}{c}E \\
{[\mathrm{GeV}]}\end{array}$ & $\begin{array}{c}Q_{6} \\
{[\mathrm{nC}]}\end{array}$ & $\begin{array}{c}\sigma_{z} \\
{[\mathrm{~mm}]}\end{array}$ & $\begin{array}{c}\gamma \varepsilon_{x} / \gamma \varepsilon_{y} \\
{[\mathrm{~mm} \mathrm{mrad}]}\end{array}$ \\
\hline drive & 1.2 & $3.2 \ldots 4.8$ & 0.55 & $30 / 3$ \\
witness & 1.2 & -2.6 & 0.55 & $30 / 3$ \\
\hline
\end{tabular}

Table 1: Beam parameters for the ASSET experiment.

structure under test. The drive bunch was dumped immediately whereas the witness travelled down the SLC linac. The wakefield seen by the witness bunch was inferred from the change in its downstream orbit. Basic beam parameters are given in Table 1. The setup is sketched in Fig. 1. The prototype DDS used in the experiment consists of 206 cells and operates in $2 \pi / 3$ mode at $11.424 \mathrm{GHz}$ (X-Band). The lowest dipole mode band is roughly Gaussianly detuned with a mean of $15.1 \mathrm{GHz}$ and a width of $2.9 \%$. The damping is provided by coupling the cells to four manifolds that run along the structure. With these features the wakefield amplitude falls below $1 \mathrm{~V} / \mathrm{pC} / \mathrm{mm} / \mathrm{m}$ within $1.4 \mathrm{~ns}$ and does not recohere above that level afterwards.

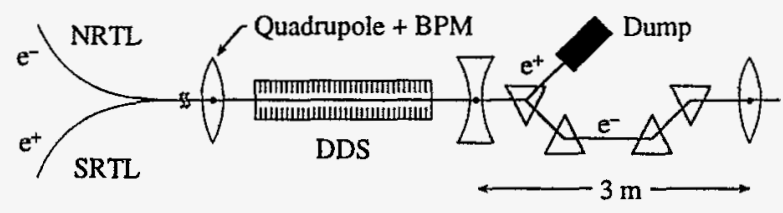

Figure 1: Layout of the ASSET facility.

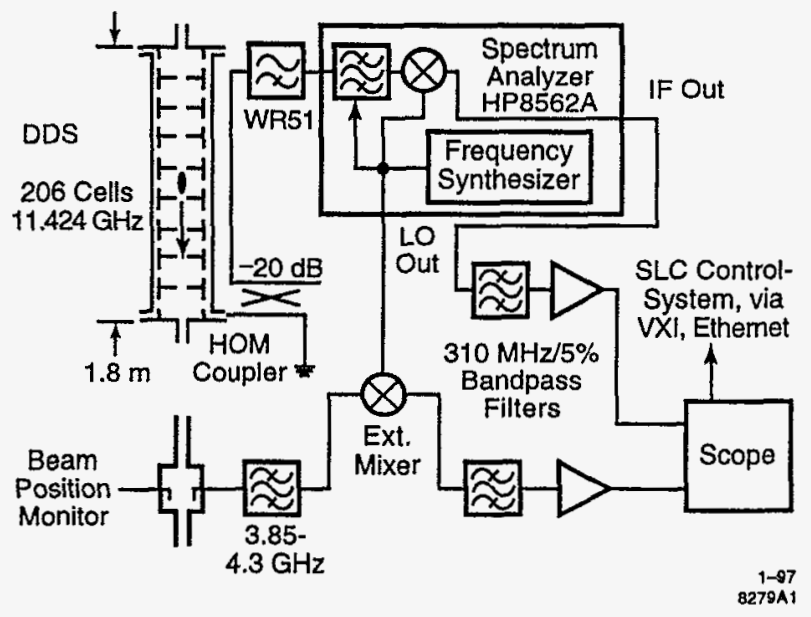

Figure 2: Electronic setup for the detection and processing of the DDS manifold signals.

Besides providing damping the manifolds permit to access beam induced dipole mode signals that origin throughout the structure. The signals from one manifold are processed in the circuit depicted in Fig. 2. With this setup it is possible to measure either the RF power excited by the beam as a function of frequency, or to select a $15 \mathrm{MHz}$ wide slice of the spectrum and determine phase and amplitude of the signal at this particular frequency. In the latter case a broad band BPM signal is used as phase reference in 
a second mixer arm. The witness deflection measurement capability of the ASSET setup provides the unique opportunity to center the beam based on dipole signals and to confirm the precision of the alignment independently with the wakefield measurement.

\subsection{Beam Based Structure Straightness Measurement}

The detuning of the structure results in a longitudinal localization of the beam-structure interaction for certain dipole mode frequencies [11]. Qualitatively this can be understood from Fig. 3 that shows a Brillouin diagram of the phase advance per cell as a function of frequency for five different longitudinal locations in the structure. When the phase advance reaches that of a free space wave it interacts coherently with the beam that moves at nearly the speed of light. The corresponding frequencies can be read from the diagram by determining the crossover of the curve with the speed-of-light-line.

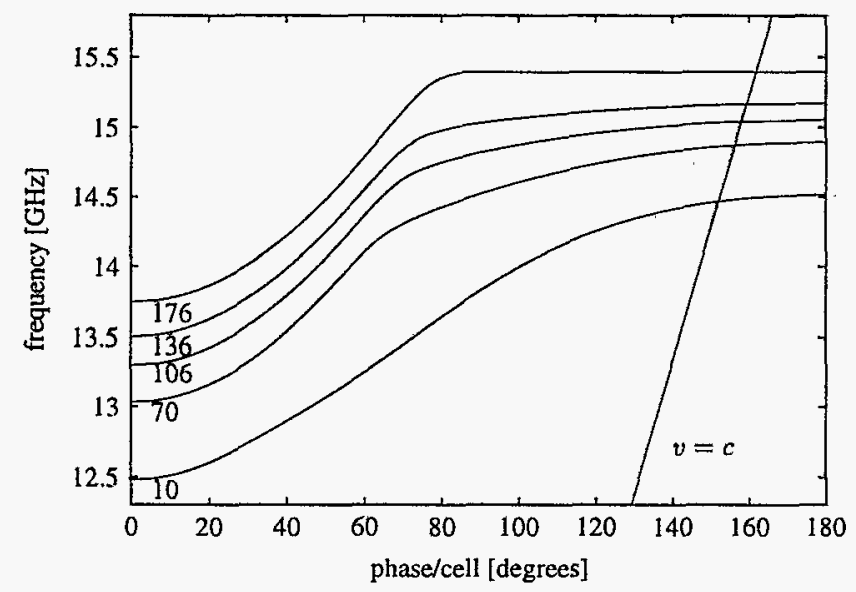

Figure 3: Brillouin diagram for the lowest dipole mode band, for five representative cells in the structure (cell numbers given).

This localization allows one to extract the cell to cell straightness of the structure from the beam induced manifold signals. For this purpose the beam was moved stepwise transversely across the structure and a complete spectrum of the manifold signal was acquired at each step. Then the two dimensional array of dipole mode power $P$ as a function of frequency $\nu$ and beam position $y$ was fit slicewise to parabolas:

$$
P\left(\nu_{n}, y_{k}\right)=A_{n}^{2}\left(y_{k}-y_{n}^{\min }\right)^{2}+B_{n}^{2}
$$

where $y_{k}$ is the beam position in the $k$ 'th step, and $A_{n}$, $B_{n}$ and $y_{n}^{\min }$ are the parabolic fit parameters for frequency slice $\nu_{n}$. A measurement is shown in Fig. 4.

Several methods to transform the minimum power positions into cell offsets have been investigated. One possibility is to describe the structure by a two-band coupled circuit model and to express the field energy deposited by a passing beam in terms of an eigenfunction expansion of TM-like modes. After minimizing this expression with respect to the beam position one obtains a system of coupled linear
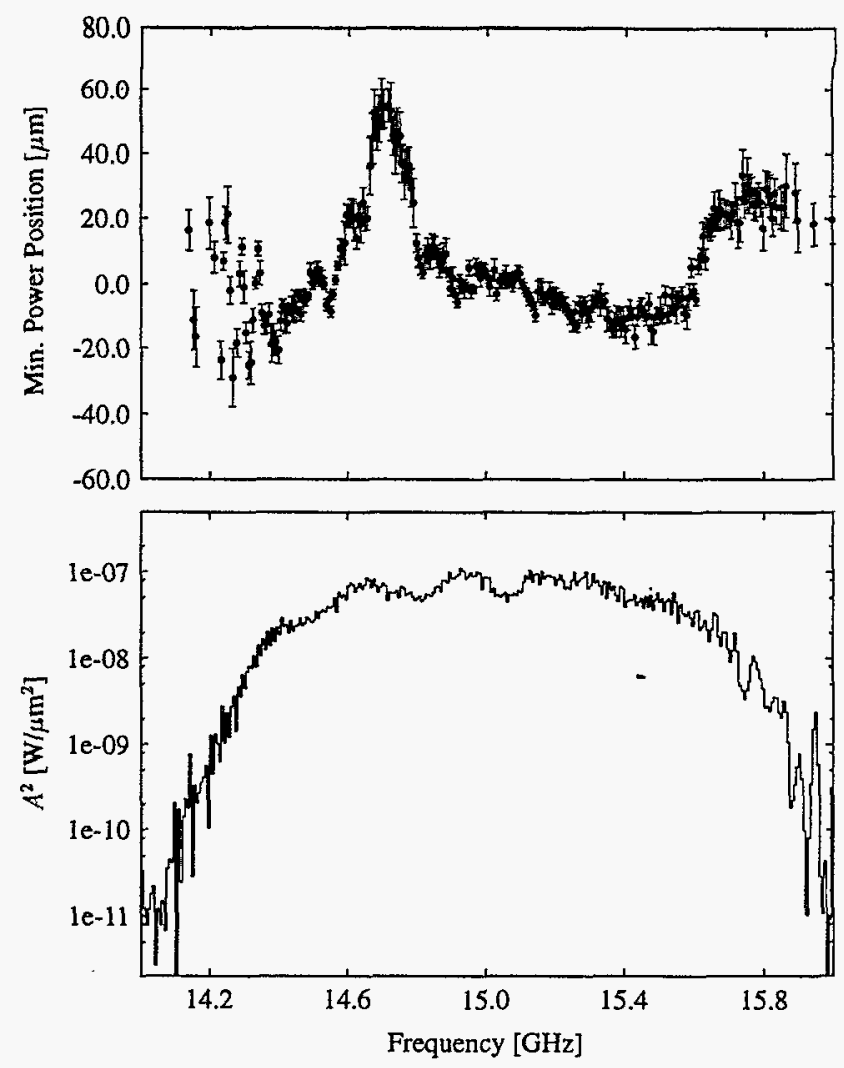

Figure 4: Minimum power positions $y^{\min }$ (upper plot) and fit parameter $A^{2}$ from Eq. (1) (lower plot) as a function of frequency for a beam position scan in the vertical plane. Note the zero position in the upper plot is arbitrarily defined.

equations that can be solved for the cell positions [12]. A different scheme that takes the manifold damping into account uses a spectral function method [11] to predict the beam induced power directly at the manifold ports for any frequency. Again one obtains a set of linear equations after minimizing the power with respect to the beam positions. Simulations indicate that also a simple one-to-one mapping of frequencies to cell positions gives a satisfactory result. A computation of the cell positions using the eigenfunction expansion is shown in Fig. 5 together with a mechanical measurement of the structure. Though the beam based measurement reproduces the basic features in the alignment curve, there are larger differences at the ends. These deviations are probably caused by thermal expansion of the material due to the raised temperature levels in the SLC tunnel. The large vertical displacement after cell 45 is due to slippage during bonding of the structure cells.

A practical application of this beam based straightness measurement is to find large internal misalignments in structures without time consuming surveying. Furthermore the knowledge of the cell to cell alignment permits to compute an optimum trajectory in the structure for minimal emittance growth. Though the excitation of wakefields in a structure with misalignments cannot be avoided in general, it is possible to minimize single bunch effects. We note that 


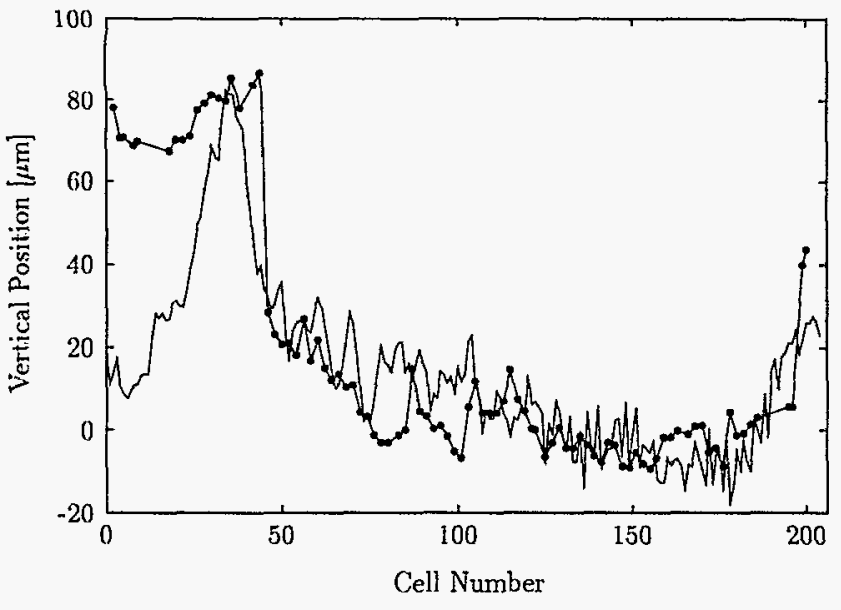

Figure 5: Cell to cell alignment, inferred from the minimum power position measurement (solid line), compared to a mechanical straightness measurement (dots).

the short range kick in cell $n$ for times of the order of the bunch length $t \approx \sigma_{z} / c \ll \lambda / c$ can be parametrized as [6]:

$$
\Delta \theta_{n}(t) \propto \frac{y_{0}+\theta_{0} s_{n}-y_{n}^{\text {cell }}}{a_{n}^{3.5}} t
$$

were $y_{0}, \theta_{0}$ are beam offset and angle with respect to the structure center and $s_{n}, a_{n}$ are the longitudinal position and iris radius of cell $n$. The emittance growth of the bunch is minimized when the slopes of integrated kick and projected beam displacement at the structure center vanish:

$$
\sum_{n} \Delta \dot{\theta}_{n}=0, \quad \sum_{n} \Delta \dot{\theta}_{n} s_{n}=0 .
$$

For known cell offsets the equations ( 3 ) can be solved for $y_{0}, \theta_{0}$ and determine thereby the optimum trajectory:

$$
\begin{aligned}
\theta_{0}=\frac{c_{1} m_{0}-c_{0} m_{1}}{m_{0} m_{2}-m_{1}^{2}}, & y_{0} & =\frac{c_{0} m_{2}-c_{1} m_{1}}{m_{0} m_{2}-m_{1}^{2}} \\
m_{\nu}=\sum_{n} \frac{s_{n}^{\nu}}{a_{n}^{3.5}}, & c_{\nu} & =\sum_{n} \frac{y_{n}^{\text {cell }} s_{n}^{\nu}}{a_{n}^{3.5}}
\end{aligned}
$$

In a large scale linac, however, performing this procedure for each structure will be too time consuming. With straight structures it is sufficient to center the beam at two longitudinal locations as described in the next section.

\subsection{Two Mode Beam Steering and Residual Beam Offset Measurement}

As noted above the ASSET setup provides one the opportunity to center the drive beam in the structure by minimizing dipole mode microwave signals, and to confirm the alignment afterwards by adding the witness beam and measuring its deflection. The kick the witness bunch receives is given by

$$
\Delta \theta_{y}(t)=\frac{e Q_{d} L_{s} W_{\perp}(t)}{E_{w}} \Delta y_{d}
$$

were $L_{s}=1.8 \mathrm{~m}$ is the length of the structure, $E_{v}$ is the energy of the witness bunch, $W_{\perp}$ the transverse wakefield, $Q_{d}, \Delta y_{d}$ drive bunch charge and offset. Thus the transverse wakefield as a function of time can be inferred by measuring the kick per unit drive bunch offset while varying the distance between the two bunches (Fig. 6). Once the wakefield amplitude is known one can also infer an unknown beam offset from the measured kick. For small beam offsets the transverse wakefield kick will scale down whereas the longitudinal wakefield at $11.4 \mathrm{GHz}$ (beam-loading) stays strong. This can introduce a systematic error for the measurement of the transverse kick due to dispersive trajectory variations. In order to suppress this effect for precise offset measurements the relative timing of the two bunches was varied in three steps at which the $11.424 \mathrm{GHz}$ beam-loading was zero, but the transverse wakefield varied nearly maximally (Fig. 6). Amplitude and phase detection of the dipole mode signal was used for centering the beam (Fig. 7). In order to align the beam both in position and angle, two modes, located near the input and output ends of the structure, were minimized simultaneously by switching the spectrum analyzer back and fourth between two frequencies. This procedure has been performed several times using different frequencies. After each attempt at centering the drive beam, the witness beam was introduced and its deflection was measured ten times at the three bunch distances. The amplitude of the kick oscillation was determined by fitting a sine-function to the three values and then converted into an equivalent drive beam offset.

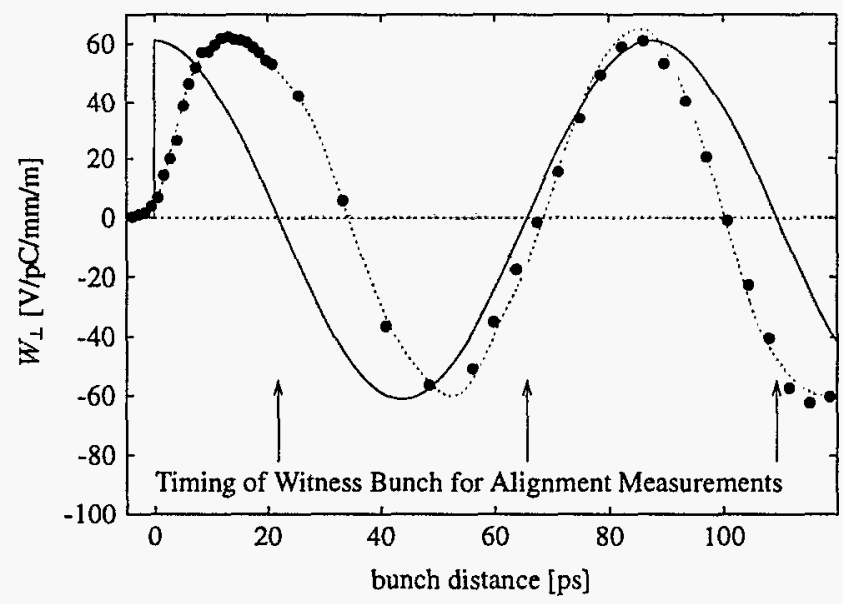

Figure 6: Transverse wakefield inferred from the deflection of the witness bunch for large drive beam offsets as a function of time (dots), a scaled prediction (dotted line) and the expected variation of the fundamental mode component (solid line, arb. units). For alignment measurements the wakefield curve was sampled at the indicated three points.

However, when analyzing the data it became clear that the relative ratios of the three measured wakefield amplitudes did not scale as the expected sine-like curve in Fig. 6. In order to fit the points one has to allow either the frequency of the oscillation to deviate from $15.1 \mathrm{GHz}$ or its 

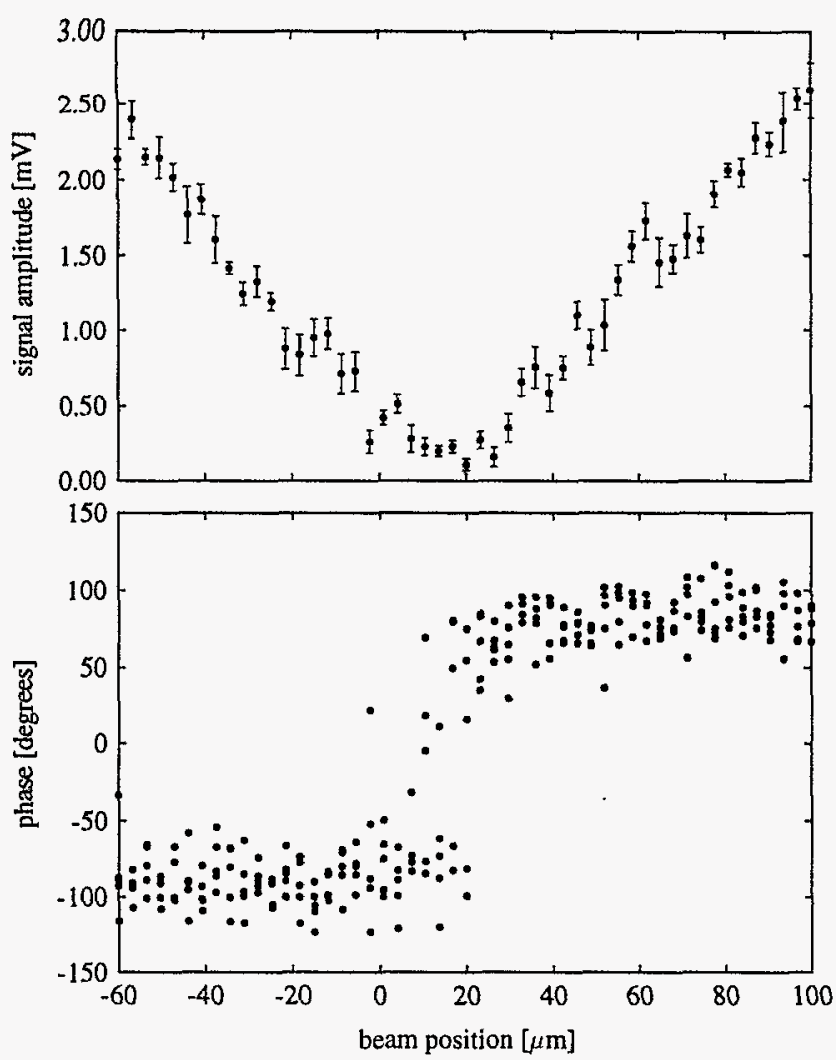

Figure 7: Amplitude and phase of the dipole mode signal at a frequency of $14.9675 \mathrm{GHz}$ as a function of the vertical beam position. For each beam position 5 pulses have been measured. The vertical beam jitter amounts to $15 \mu \mathrm{m}$ rms.

phase with respect to the bunch crossing to deviate from $0^{\circ}$. Fig. 8 shows the measurement results when we allow the frequency to vary. The pattern of the data could be related to the relatively large internal misalignments in the structure. If the beam is centered on average it will excite modes with opposite signs in different regions of the structure that beat against each other. As pointed out above it is now impossible to avoid the excitation of wakefields for any trajectory. We define the optimum beam position as the point where the slope of the wakefield for $t \rightarrow 0$ vanishes. In our measurements, however, we sample the beating oscillation at only three points and interpret it as a pure harmonic oscillation for which we determine amplitude and frequency. Apparently we observe the inferred frequency going down for small amplitudes, whereas at some point it jumps over to values above $15.1 \mathrm{GHz}$. The solid line in Fig. 8 is an analytical prediction which is based on the mechanical alignment measurement. This curve exhibits the same qualitative behavior as the measurement but not the observed magnitude. The dashed curve, which fits the data better, assumes in addition a sine-like transverse force at the fundamental mode frequency $11.424 \mathrm{GHz}$ with an amplitude corresponding to $10 \mu \mathrm{m}$ beam offset. Such a component could be generated by specific cell misalignments although its magnitude has not yet been estimated.

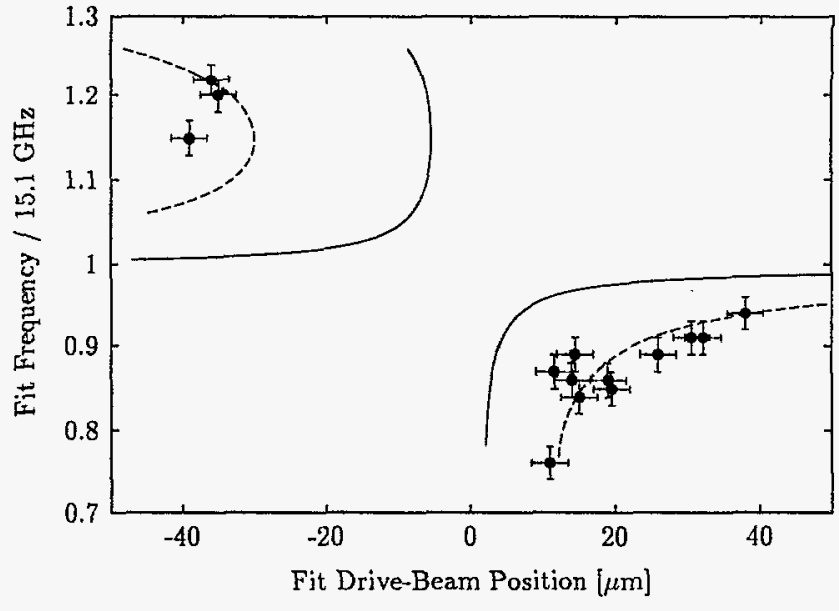

Figure 8: Inferred frequencies and equivalent beam offsets for several trials of centering the drive beam.

\section{SUMMARY}

Dipole mode based beam steering is a promising technique to control transverse wakefields in accelerating structures. It will be essential to push performance limitations in future low emittance or high current linear accelerators. The comparison of experiences from the SLC and the modern DDS structure shows that dedicated HOM couplers are needed to distinguish both transverse polarizations of the modes. The DDS manifolds permit to access all modes along the structure which makes it possible to perform an in-situ beam based straightness measurement. Using phase and amplitude detection an alignment precision of better than $40 \mu \mathrm{m}$ has been achieved and verified by measuring the wakefield deflection of a second test bunch.

The author is indebted to C.Adolphsen, R. Assmann, K. Bane, P.Emma, R. Jones, N. Kroll, R. Miller, C. Nantista, T.Slaton, J. Wang, D. Whittum for contributions to the presented results. This work was supported by the Department of Energy, contract DE-AC03-76SF00515.

\section{REFERENCES}

[1] G. Loew, R. Helm, H. Hoag, R. Koontz, R. Miller, Conf. on High Energy Part. Accel., Yerevan (1969)

[2] R.H.Miller et al. Linac 96, Geneva (1996)

[3] N. Kroll, these proceedings

[4] M.Dohlus et al. DESY 96-169 (1996)

[5] T. Shintake, Jpn. J. Appl. Phys. 31, 1567 (1992)

[6] R. Palmer, Ann. Rev. Nucl. Part. Sci. 40, 529 (1990)

[7] R. Assmann et al. these proceedings

[8] M. Seidel, C. Adolphson, R. Assmann, D.H. Whittum, these proceedings

[9] C. Adolphsen et al. Phys. Rev. Lett. 74, 2475 (1994)

[10] C. Adolphsen et al., subm. to Phys. Rev. Lett. (1997)

[11] R.M. Jones et al., these proceedings

[12] M. Seidel et al. subm. to Phys. Rev. Lett. (1997) 